EISSN: 2706-7939 ISSN: 2077-4508

DOI: 10.36632/ije/2021.10.2.10

Journal homepage: www.curresweb.com

Pages: 103-108

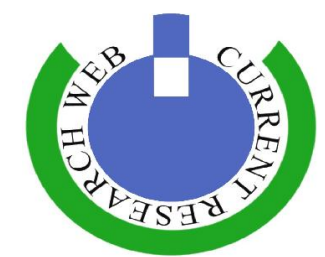

\title{
Detection of Biofilm formation among Staphylococcus aureus isolated from hospital- admitted patients' wounds and their relationship with antibiotic resistance
}

\author{
Abu baker H. Abduelrhman \\ Department of Medical Laboratory Technology, High Institute of Science and Medical technology, EL- \\ Garahbolii. Libya
}

Received: 10 May $2021 \quad$ Accepted: 15 June $2021 \quad$ Published: 20 June 2021

\begin{abstract}
Background: Biofilm is known to be formed by microorganisms. The biofilms are commonly found in chronic wound infections, surgical site infections, implants, and other places. They can cause recalcitrant infections and are known to have high antibiotic resistance. The aim of this research was to prevalence and compare the in vitro biofilm-forming ability of Staphylococcus aureus isolated from wounds of hospitalized patients', as well as their association with antibiotic resistance. Materials and methods: Using standard microbiological techniques, 57 clinical isolates of $S$. aureus were obtained from 128 wounds samples. $S$. aureus was identified by VITEK2 system. Tissue culture plate (TCP) analysis was used to detect biofilm formation in these isolates. The modified Kirby-Bauer disk diffusion method was used to test antibiotic sensitivity in accordance with Clinical and Laboratory Standards Institute guidelines. The modified Kirby-Bauer disk diffusion method was used to test antibiotic sensitivity in accordance with Clinical and Laboratory Standards Institute guidelines. Results: $S$. aureus was identified by VITEK2 system The Biochemical characteristics and antibiotics resistance by VITEK2 system with excellent probability $99 \%$. Biofilm formation occurs in $26(31,32 \%)$ of $S$. aureus isolates using the TCP method. When compared to biofilm nonproducers, biofilmproducing $S$. aureus had a higher incidence of antimicrobial resistance $(P<0.05)$. Conclusion: An in vitro method revealed that $S$. aureus isolated from hospitalized patients' wound infections has a high degree of biofilm-forming ability. Antibiotic resistance is highly prevalent in biofilm-producing strains.
\end{abstract}

Keywords: Staphylococcus aureus, biofilm, Antibiotic resistance, in vitro

\section{Introduction}

The aggregation of bacteria in a self-produced extracellular matrix of exopolysaccharides (EPSs), proteins, and some micromolecules such as DNA is known as a biofilm. They can grow on biotic as well as abiotic surface (Gowrishankar et al., 2012).

Biofilm is an important virulence factor that provides a safe environment for organisms to survive (Shashikala et al., 2001). Biofilm-associated infections are difficult to treat because the biofilm confers resistance against the host immune system and renders the bacterial cells antibiotic-resistant (Høiby et al., 2010).

S. aureus is an opportunistic pathogen that has been linked to the majority of skin and soft tissue infections. It can be found in the nasopharynx, skin, eyes, and intestine (Ziebuhr et al., 2001) In $S$. aureus isolates, biofilm formation occurs via polysaccharide intercellular adhesion (PIA) as well as microbial surface components that recognize adhesive matrix molecules (MSCRAMMs) (Andhale et al., 2016). These structures mediate $S$. aureus initial attachment to both host tissues and biomaterials (Hassan et al., 2005). Biofilm formation disrupts the innate immune system's bacterial recognition and killing mechanisms (Andhale et al., 2016).

Corresponding Author: Abu baker H. Abduelrhman, Department of Medical Laboratory Technology, high institute of science and Medical technology, EL-Garahbolii. Libya.

E-mail: hmeda63@gmail.com 
Antibiotic resistance mechanisms also include delayed antimicrobial agent penetration through the biofilm matrix, altered growth rates of biofilm forming organisms, and other physiological changes that occur during biofilm growth. Many, and chronic recurrent infections (Hassan et al., 2005).

As a result, our objective is to investigate the ability of $S$. aureus isolated from hospitalized patients' wounds to form biofilms in vitro and their relationship to antibiotic resistance

\section{Materials and Methods}

\section{Preparation of clinical pus.}

The cross-sectional observational research was performed out at the Department of medical technology at higher institute of science and technology. A total of 128 routine pus samples were collected from wounds at various body sites patients of Msallata hospitals from March 2019 to July 2019. Approximately, $0.5 \mathrm{~g}$ of wound samples were resuspended in to phosphate-buffered saline (PBS) with $5 \mathrm{ml}$ of $10 \%(\mathrm{w} / \mathrm{v})$ in PBS $(0.01 \mathrm{M}$ Tris solution $(\mathrm{pH} 7.5), 14.5 \mathrm{mM}, \mathrm{NaCl}$ and $10 \mathrm{mM}, \mathrm{CaCl} 2)$. wound solutions were vortexed and clarified by centrifugation at $3000 \mathrm{rpm}$ for $10 \mathrm{~min}$. The clarified supernatant $(1.5-2.0 \mathrm{ml})$ was collected, and store at $4-8^{\circ} \mathrm{C}$ for short term storage until use.

\section{Detection of $S$. aureus by free coagulase:}

Coagulase causes plasma to clot by converting fibrinogen to fibrin. Tested by: Slide coagulase test (to detect bound coagulase).One hundred microliters of brain heart infusion broth culture of each tested isolate was added to $0.5(\mathrm{ml})$ of 5 -fold dilution fresh citrated rabbit plasma. The tubes were incubated at $35-37^{\circ} \mathrm{C}$ and examined for clotting after $1,2,3$, and $24 \mathrm{~h}$. The results were interpreted as follows: Score of $2+$ (small organized clot), $3+$ (large organized clot), $4+$ (entire content of the tube coagulates and is not displaced when the tube is inverted).The results were considered positive evidence of coagulase production.

\section{Isolation and enumeration of $S$. aureus (FDA, 2002).}

Isolation and enumeration of $S$. aureus in was carried out by spreading $0.1(\mathrm{ml})$ of each sufficient dilution onto the surface agar. Baird parker media (Oxoid; CM0275) supplemented with egg yolk and potassium tellurite solution. Plates were incubated at $37^{\circ} \mathrm{C}$ for $48 \mathrm{~h}$, then the presence of typical colonies, which appears gray-black, shiny and convex with a narrow white margin surrounded by a clearing zone. The numbers of typical colonies were counted. Suspected $S$. aureus were isolated by culturing on brain heart infusion agar (Oxoid;CM225) slants medium for further identification.

\section{Purification and identification of $S$. aureus.}

S. aureus colonies obtained from all previously mentioned media were chosen and picked up according to variation in culture characteristics and colony formation then purified by streak-plate method on Nutrient agar medium. Pure isolates were maintained on slants of the same medium at $4^{\circ} \mathrm{C}$ for subsequent identification. $S$. aureus was identified by VITEK 2 system for identification were carried out according to Funke et al. (1998).

The reagent cards have 64 wells that can each contain an individual test substrate. Substrates measure various metabolic activities such as acidification, alkalinization, enzyme hydrolysis, and growth in the presence of inhibitory substance. There are currently four reagent cards available for the identification of different organism classes as follows:

\section{Suspension preparation:}

A disposable bacterial needle used to transfer a single colony of a pure culture and suspended in $3.0 \mathrm{ml}$ of sterile saline (aqueous $0.45 \%$ to $0.50 \% \mathrm{NaCl}, \mathrm{pH} 4.5$ to 7.0 ) in a $12 \times 75 \mathrm{~mm}$ clear plastic (polystyrene) test tube. The turbidity was adjusted according Table (1) and measured using a turbidity meter called the DensiChek.

Identification cards were inoculated with S.aureus suspensions using an integrated vacuum apparatus. A test tube containing $S$. aureus suspension was placed into especial rack (cassette) and the identification card was placed in the neighbor in gloat while inserting the transfer tube into the corresponding suspension tube 
Table 1: Suspension turbidities used for card inoculation

\begin{tabular}{cc}
\hline Product & McFarland Turbidity Range \\
\hline GN & $0.50-0.63$ \\
GP & $0.50-0.63$ \\
YST & $1.80-2.20$ \\
BCL & $1.80-2.20$ \\
\hline
\end{tabular}

\section{Tissue Culture Plate method}

Christensen et al. (1985) described this quantitative gold standard method for biofilm detection. In a nutshell, a $S$. aureus colony was isolated from a fresh agar plate and inoculated in $2 \mathrm{~mL}$ of trypticase soy broth. Overnight at $37^{\circ} \mathrm{C}$, the broth was incubated. With fresh medium, the culture was diluted to 1:100. $200 \mathrm{~L}$ of the diluted culture was poured into a sterile individual plate with 96 flat-bottom polystyrene wells. In the same way, the control organisms were processed.

The plate was incubated for 24 hours at $37^{\circ} \mathrm{c}$. The contents of each well were gently tapped out after incubation. To remove free-floating bacteria, the wells were washed with $200 \mathrm{~L}$ of phosphate buffer saline ( $\mathrm{pH}$ 7.3). Biofilms formed by bacteria attached to the wells were fixed with 99 percent methanol and stained with 0.1 percent crystal violet (CV). The excess stain was gently washed away, and the plate was set aside to dry. A micro ELISA auto-reader (HUMAN) was used to measure the optical density of the stained adherent biofilm at a wavelength of $570 \mathrm{~nm}$.

The experiment was carried out three times. Biofilm production was interpreted using the criteria described by Stepanovic et al. (2007), and bacteria were classified as biofilm nonproducers, weak, moderate, or strong biofilm producers.

\section{Antimicrobial Sensitivity}

The clinical isolates were tested for antibiotic sensitivity using the standard disk diffusion technique (modified Kirby-Bauer method) in Müller-Hinton agar (MHA), and the results were interpreted according to Clinical and Laboratory Standards Institute guidelines (CLSIG 2002). Antibiotics that were tested included: gentamicin $(10 \mu \mathrm{g})$, ciprofloxacin $(5 \mu \mathrm{g})$, rifampicin $(5 \mu \mathrm{g})$, vancomycin $(30 \mu \mathrm{g})$, erythromycin $(15 \mu \mathrm{g})$ and cotrimoxazole $(25 \mu \mathrm{g})$ (HiMedia Laboratories, Mumbai, Maharashtra, India). S. aureus ATCC 25923 was used as the control organism.

\subsection{Statistical analysis}

The Chi-square analysis was used to measure categorical data. A $P$-value of 0.05 was considered statistically significant.

\section{Results}

\subsection{S. aureus identification:}

S. aureus was incidence in clinical pus and isolated and enumerated onto plates of blood media Shaped of colony were Raised, circular, entire. Biochemical Identification of isolates which belonging to $S$. aureus showed Smooth Texture, Golden yellow Pigmentation, Shape Cocci in clusters \& pairs, no motility, Beta Hemolysis on blood agar, facilitative anaerobic, positive Gram, positive Catalase, Coagulase and Urease negative Oxidase. On blood agar plates, colonies of $S$. aureus often cause $\beta$ hemolysis. S. aureus formed large yellow colonies surrounded by wide yellow zones and turned the color of the medium from pink to yellow. S. aureus was a Gram-positive spherical bacterium approximately $1 \mu \mathrm{m}$ in diameter and formed grape-like clusters.

The Biochemical characteristics of $S$. aureus was confirmed with excellent probability $99 \%$ after full Biochemical identification by VITIK2 system as well as the susceptibility information was provided as shown in table ( 2 ).

\subsection{Biofilm formation}

Among some of the 57 clinical isolates of $S$. aureus obtained from the wounds, biofilm formation was observed in $26(45.61 \%)$ isolates tested Only five isolates $(19.23 \%)$ were classified as strong biofilm producer, while eight $(30.76 \%)$ of the clones were classified as moderate producers. More than 
half of the producers (50\%) were found weak biofilm producers. A total of $31(54.38 \%)$ of the samples showed no evidence of biofilm producers (table 3 ).

Table 2: Biochemical characteristics of: S. aureus by VITEK 2

\begin{tabular}{|c|c|c|c|c|c|c|c|c|c|c|c|}
\hline \multicolumn{3}{|c|}{ Identification Information } & & \multicolumn{4}{|c|}{ Analysis Time: 4.58 hours } & \multicolumn{4}{|c|}{ Status: Final } \\
\hline \multirow{2}{*}{\multicolumn{3}{|c|}{ Selected Organism }} & & \multirow{2}{*}{\multicolumn{3}{|c|}{$\begin{array}{c}\text { 99\% Probability: } \\
\text { Bionumber } \\
\end{array}$}} & & \multirow{2}{*}{\multicolumn{4}{|c|}{$\begin{array}{l}\text { Staphylococcus aureus } \\
\mathbf{0 1 0 4 0 2 0 6 2 6 6 3 2 3 1}\end{array}$}} \\
\hline & & & & & & & & & & & \\
\hline AMY & - & PIPLC & - & $\mathrm{dXYL}$ & - & ADH1 & + & BGAL & - & AGLU & - \\
\hline APPA & - & CDEX & - & AspA & - & BGAR & - & AMAN & - & PHOS & + \\
\hline LeuA & - & ProA & - & BGURr & - & AGAL & - & PyrA & + & BGUR & - \\
\hline AlaA & - & & - & dSOR & - & URE & - & POLYB & + & dGAL & + \\
\hline dRIB & - & lLATk & + & LAC & - & NAG & - & dMAL & + & BACI & + \\
\hline NOVO & - & NC6.5 & + & dMAN & + & $\mathrm{dMNE}$ & + & MBdG & + & PUL & - \\
\hline dRAF & - & O129R & + & SAL & - & SAC & + & dTRE & + & $\mathrm{ADH} 2 \mathrm{~s}$ & - \\
\hline OPTO & + & Installed & ITE & ( ) Syster & $\mathrm{Ve}$ & on: 08.01 & & & & & \\
\hline
\end{tabular}

Table 3: The MTP method was used to analyze biofilm production.

\begin{tabular}{ccc}
\hline Degree of biofilm formation & No. of $\boldsymbol{S}$. aureus $\mathbf{( N = 5 7 )}$ & Percentage \\
\hline Strong & 5 & 19.23 \\
Moderate & 8 & 30.76 \\
Weak & 13 & 50 \\
None & 31 & 54.38 \\
\hline
\end{tabular}

\subsection{Antibiotic sensitivity of $S$. aureus isolate}

As a result, the aim of the research at the pattern of antibiotic resistance, when compared to biofilm producing to non-producers was associated with a higher incidence of antimicrobial resistance, as shown in table 3, gentamicin ( $53.84 \%$ vs $6.45 \% \mathrm{P}=0.0007$ ), ciprofloxacin ( $80.76 \%$ vs $9.67 \%$ $\mathrm{P}=0.0001$ ), ampicillin ( $92.30 \%$ vs $35.48 \% \mathrm{P}=$ ), erythromycin ( $\%$ vs $\% \mathrm{P}=0.00011$ ) and cotrimoxazole ( $7.69 \%$ vs $3.10 \% \mathrm{P}=0.049)$ (Table 4$)$.

Table 4: Comparison of antibiotics resistance pattern in biofilm-positive and biofilm-negative S. aureus

\begin{tabular}{|c|c|c|c|}
\hline Antibiotics & BP $(N=26)$ & BN $(\mathrm{N}=31)$ & P. value \\
\hline Gentamicin $10 \mu \mathrm{g}$ & $14(53.84)$ & $2(6.45)$ & $* 0.0007$ \\
\hline Ciprofloxacin $5 \mu \mathrm{g}$ & $21(80.76)$ & $3(9.67)$ & $0.0001 *$ \\
\hline Rifampicin $5 \mu \mathrm{g}$ & $0(0)$ & $0(0)$ & NA \\
\hline Vancomycin $30 \mu \mathrm{g}$ & $0(0)$ & $0(0)$ & NA \\
\hline Ampicillin $10 \mathrm{~g}$ & $24(92.30)$ & $11(35.48)$ & $0.00011 *$ \\
\hline Erythromycin $15 \mu \mathrm{g}$ & $11(42.30)$ & $1(3.10)$ & $0.0003 *$ \\
\hline Cotrimoxazole $25 \mu \mathrm{g}$ & $2(7.69)$ & $1(3.10)$ & $0.049^{*}$ \\
\hline
\end{tabular}

Abbreviation: BP. Biofilm positive, BN. biofilm negative

Note: Statistically significant $(\mathrm{P}<0.05)$

NA, no applicable.

\section{Discussion}

$S$. aureus is a zoonotic pathogen that can cause a wide range of symptoms in both animals and humans. S. aureus that produces biofilms is a major public and animal health concern. The identification of $S$. aureus adherence property has revealed associations between biofilm formation and degree of pathogenicity, with the organism's virulence property found to vary with its ability to adhere to the surface (Baddour et al., 1984).

In this study, we discovered that $S$. aureus isolated from hospitalized patients' wounds can form biofilms in vitro and that this ability is associated with drug resistance. TCP method in vitro screening procedures were used to assess the ability of 57 S.aureus isolates to form biofilm.

In the present research, the prevalence of biofilm production was higher $(45.61 \%)$ percent detected by TCP. Our findings, which were based on wound samples, are similar to those of a previous study, which found that $50 \%$ of blood samples formed biofilms (El-Nagdy et al., 2020). Biofilm formation is influenced by a variety of factors, including the environment, nutrient availability, geographical origin, 
specimen types, surface adhesion characteristics, and the organism's genetic makeup (Poudel et al., 2015).

As a result, the adherence property of biofilm producers was classified as strong, moderate, or weak. In the current study, (19.23\%) of the $S$. aureus were highly virulent and exhibited high adherence. Our findings were consistent with those of another Algerian study (Lotfi et al., 2014 ).

showed five (19.23\%) strongly adherent, eight (30,76 \%) moderately adherent, haif (50\%) weakly adherent, and 31 (68.84\%) nonadherent isolates (Lotfi et al., 2014 ).

Because bacteria in biofilms are resistant to antibiotic compounds, biofilm infections are clinically significant (Doebbeling, 1995). S. aureus that produces biofilms was more prevalent. Biofilm producers are more resistant to antimicrobials than nonproducers of biofilms (Mathur et al., 2006).

Biofilm producers were resistant to erythromycin, gentamicin, tetracycline, chloramphenicol, and teicoplanin in our research, whereas none of the biofilm nonproducers were $(P<0.05)$. Biofilm producers had significantly higher rates of resistance to cotrimoxazole and ciprofloxacin than nonproducers $(P<0.05)$. It has previously been reported that Gram-positive bacteria that produce biofilms are more resistant to erythromycin, cotrimoxazole, and ciprofloxacin (Hassan et al., 2011) and (Mishra et al., 2014). Our findings corroborate those of a previous study conducted (Ansari et al., 2014; Neopane et al., 2018).

As a result, antibiotic resistance was found to be higher among biofilm-producing $S$. aureus than among nonproducers in the current study. These findings suggest that biofilm formation could be a key factor in increasing resistance to commonly used antibiotics. Our results are consistent with those of a prior study (Neopane, et al., 2018).

Vancomycin is reported to be the most effective antibiotic for Gram-positive bacteria accordingly, our data also showed that all the isolates including biofilm producers were sensitive to vancomycin which is similar to others studies (Harika et al., 2020 and Neopane et al., 2018).

\section{Conclusion}

One of the most important virulence factors of $S$. aureus is biofilm production, which allows them to resist or inhibit the effects of various antibiotics. vancomycin is the best antibiotics to use against $S$. aureus in wound infections. As a result, we recommend that biofilm formation and antibiotic resistance profiles in S. aureus wound isolates be monitored on a regular basis. This may aid in the formulation of an effective antimicrobial policy for the treatment of wound infection in the early stages.

\section{References}

Andhale, J.D., R.N. Misra, N.R. Gandham, K.M. Angadi, S.V. Jadhav, C.R. Vyawahare, et al., 2016. Incidence of Pseudomonas aeruginosa with special reference to drug resistance and biofilm formation from clinical samples in tertiary care hospital. J. Pharm. Biomed. Sci., 6: 38791.

Ansari, S., H.P. Nepal, R. Gautam, et al., 2014. Threat of drug resistant Staphylococcus aureus to health in Nepal. BMC Infect. Dis., 14:157.

Baddour, L.M., G.D. Christensen, M.G. Hester, and A.L. Bisno, 1984. Production of experimental endocarditis by coagulase-negative staphylococci: variability in species virulence. J Infect Dis., 150(5):721-727.

Baird, D., 1996. Staphylococcus: cluster forming gram positive cocci. In: Collee JG, Fraser AG, Marmion BP, Simmons A, editors. Mackie and McCartney Practical Medical Microbiology. 14th ed. Vol. 2. London: Churchill Livingstone, 245-261.

Christensen, G.D., W.A. Simpson, J.J. Younger, et al., 1985. Adherence of coagulase-negative staphylococci to plastic tissue culture plates: a quantitative model for the adherence of staphylococci to medical devices. J. Clin. Microbiol., 22(6):996-1006.

CLSIG, 2007. Clinical and Laboratory Standards Institute. Performance standards for antimicrobial susceptibility testing; Seventeenth Informational Supplement. Document, M100-S17. Wayne, PA: CLSI.

Doebbeling, B., 1995. The epidemiology of methicillin-resistant Staphylococcus aureus colonisation and infection. J. Chemother., 7(Suppl 3): 99-103. 
El-Nagdy, A.H., G.M. Abdel-Fattah, and Z. Emarah, 2020. Detection and Control of Biofilm Formation by Staphylococcus aureus from Febrile Neutropenic Patient. Infect Drug Resist. Sep 7; 13:30913101. doi: 10.2147/IDR.S259914. PMID: 32982324; PMCID: PMC7495500.

FDA, Food and Drug Administration, 2002.Bacterio-logical analytical manual. 9th Ed., AOAC Int., Arlington, VA, USA.

Funke, Guido, et al., 1998. "Evaluation of the VITEK 2 system for rapid identification of medically relevant gram-negative rods." Journal of clinical microbiology 36.7: 1948-1952.

Gowrishankar, S., N. Duncun Mosioma, and S. Karutha Pandian, 2012. Coralassociated bacteria as a promising antibiofilm agent against methicillinresistant and susceptible Staphylococcus aureus biofilms. Evid Based Complement Alternat Med., 862374.

Harika, K., V.P. Shenoy, N. Narasimhaswamy, and K. Chawla Detection of Biofilm Production and Its Impact on Antibiotic Resistance Profile of Bacterial Isolates from Chronic Wound Infections. J. Glob Infect Dis., 12(3):129-134. Published 2020 Aug 29. doi:10.4103/jgid.jgid_150_19.

Hassan, N.S., A.S. Esmail, and A.H. Mahmoud, 2009. Bacteriological assessment of some ready-to-eat foods. Kafr El-Sheikh, Vet. Med. J. Sci. Congress, 3: 474- 487.

Hassan, A., J. Usman, F. Kaleem, M. Omair, A. Khalid, and M. Iqbal, 2011. Detection and antibiotic susceptibility pattern of biofilm producing Gram positive and Gram negative bacteria isolated from a tertiary care hospital of Pakistan. Malays J. Microbiol., 7(1):57-60.

Høiby, N., T. Bjarnsholt, M. Givskov, S. Molin, and O. Ciofu, 2010. Antibiotic resistance of bacterial biofilms. Int. J. Antimicrob Agents., 35: 322 - 332.

Lotfi, G., H. Hafida, K. Nihel, et al., 2014. Detection of biofilm formation of a collection of fifty strains of Staphylococcus aureus isolated in Algeria at the University Hospital of Tlemcen. J Bacteriol Res., 6(1):1-6.

Mathur, T., S. Singhal, S. Khan, D. Upadhyay, T. Fatma, and A. Rattan, 2006. Detection of biofilm formation among the clinical isolates of Staphylococci: an evaluation of three different screening methods. Indian J. Med. Microbiol., 24(1):25-29.

Mishra, S.K., P. Basukala, O. Basukala, K. Parajuli, B.M. Pokhrel, and B.P. Rijal, 2014. Detection of biofilm production and antibiotic resistance pattern in clinical isolates from indwelling medical devices. Curr Microbiol. 2015 Jan; 70(1):128-34. doi: 10.1007/s00284-014-0694-5. Epub Sep 20. PMID: 25239012.

Neopane, P., H.P. Nepal, R. Shrestha, O. Uehara, Y. Abiko, 2018. In vitro biofilm formation by Staphylococcus aureus isolated from wounds of hospital-admitted patients and their association with antimicrobial resistance. Int. J. Gen. Med., 11:25-32. Published 2018 Jan 18. doi:10.2147/IJGM.S153268.

Poudel, P., N. Adhikari, and P.K. Shah, 2015. Multi-drug resistant bacterial isolates associated with blood stream infection. ASRJETS., 14(2):23-52.

Shashikala, V., F. Ali, N. Lokare, and J. Matew, 2016. Diabetic foot ulcers and biofilm formation the culprits. Int. J. Biomed. Adv. Res., 7:428 33.

Stepanovic, S., D. Vukovic, V. Hola, et al., 2007. Quantification of biofilm in microtiter plates: overview of testing conditions and practical recommendations for assessment of biofilm production by staphylococci. APMIS., 115(8):891-899.

Ziebuhr, W., I. Loessner, V. Krimmer, and J. Hacker, 2001. Methods to detect and analyze phenotypic variation in biofilm-forming Staphylococci. Methods Enzymol., 336:195-205. 